\title{
Propuesta Educativa de Enriquecimiento Extracurricular y su Implementación: PEEEI
}

Krissia Morales Chacón*

\section{Resumen}

Este trabajo contiene un Plan de intervención psicoeducacional para niños superdotados con un abordaje multidimensional de acuerdo con el que la noción de inteligencia está integrada por múltiples componentes que son relativamente independientes pero que son esenciales para un desarrollo ideal de niños superdotados. Su objetivo general es implementar una propuesta educativa de enriquecimiento extracurricular - EPEEI dirigido al cuidado integral de nińos superdotados en edades tempranas, a través de su aplicación en el campo. El método utilizado se desarrolló en dos etapas. La primera etapa estaba compuesta de tres campos de intervención: a) Programas de psicología individual para incentivar a los nińos de acuerdo a sus fortalezas; b) Actividades de apoyo y capacitación para padres sobre el tema de nińos superdotados; c) Orientación y capacitación docente. La segunda etapa consistió en probar el plan durante un período de aproximadamente 6 meses. De acuerdo a los resultados, se comprobó que los niños superdotados en nuestro contexto, carecen de oportunidades psicopedagógicas que atiendan genuinamente a sus necesidades educacionales especiales. Tanto las escuelas como las familias carecen de la información y capacitación necesarias para los casos identificados. Ellos demuestran desorientación, frustración e ignorancia de los posibles servicios y recursos que podrían ofrecerles apoyo en esa tarea (Por ejemplo: Tecnologías de Información y comunicación).

Palabras-clave: Niños superdotados; Plan psicoeducacional; Implementación.

\footnotetext{
* Professora Doutora em Educação pela PUC-RS. Vice-presidente e membro do Conselho técnico da Associação Gaúcha de Apoio às Altas Habilidades/Superdotação. Porto Alegre, Rio Grande do Sul, Brasil.
} 


\section{Educational Plan of Extracurricular Enrichment and its Implementation: EPEEI}

\section{Abstract}

This work contains a Plan of Psycho-educational intervention for gifted children with a multidimensional approach, according to which the notion of intelligence is composed of multiple components, which are relatively independent but still essential for the optimal development of super-talented children. Its overall objective is to implement an educational plan for extracurricular enrichment EPEEI directed to the comprehensive care of super-talented children as at an early age, through its application in the field. The method used was developed in two stages. The first stage involved three fields of intervention: a) Individual care psychology programs to encourage children, according to their strengths. b) Support activities and training for the parents on the subject of super-talented children. c) Guidance and training to teachers. The second phase involved trying the plan for a period of approximately 6 months. According to the results, it was proven that super-talented children in our environment are lacking psycho-pedagogical opportunities that genuinely meet their special educational needs. Both schools and families, lacked the information and training necessary to address the identified cases. They demonstrated disorientation, frustration and ignorance of the possible services and resources that could offer support in this task (For example: Information and communication technologies).

Keywords: Gifted children; Plan Psycho-educational; Implementation.

\section{Introducción}

Hoy, la necesidad de trascender los paradigmas educativos igualitarios y lineales en sociedades como la nuestra, constituye un reto y una oportunidad, pues significa superar las disposiciones y prácticas establecidas en el sistema educativo convencional enfocado hacia la incorporación de los individuos a la sociedad, únicamente como "capital intelectual"; sin considerar su potencial individual y desarrollo integral: aptitudes y habilidades cognitivas, de criticidad, creatividad, motricidad, sensorialidad y sensibilidad ético-moral (espiritual, más allá de los sentidos) como seres humanos pluridimensionales.

Los paradigmas educativos emergentes promueven nuevas perspectivas y la posibilidad de experimentar y reflexionar sobre lo que se ha venido haciendo en educación; propiciando nuevas concepciones educativas donde se respete la diversidad educativa. El proyecto de investigación educativo que se presenta a continuación pretende contribuir en la solución al problema de ausencia de intervención psico-pedagógica oportuna para la niñez con superdotación en el sistema educativo regular; para ello en una primer etapa se despliega una propuesta educativa de enriquecimiento extracurricular (PEEE) para niños/as con superdotación intelectual que pretende mediante una II etapa empirica o de aplicación propiciar la estimulación oportuna y directa de 
sus potencialidades particulares, de modo que se manifiesten en habilidades y dominios, maximizando su desarrollo psico-pedagógico y calidad de vida.

El desarrollo de la presente proposición significa la posibilidad de apoyar su desarrollo integral; no sólo educativo. Bajo estos criterios, se enmarca la propuesta de intervención educativa, proponiendo un proceso científico y sistemático en el ámbito pedagógico que facilite la realización de una intervención bien dirigida y a la vez integradora.

La construcción e implementación práctica de la propuesta surgió como respuesta a los resultados obtenidos en una investigación de identificación diagnostica en niños/as en edades tempranas con posible superdotación ${ }^{1}$. Esta evidenció que aproximadamente un $2.29 \%$ de los niños y niñas de la muestra seleccionada (más de 600 niños/as) poseen una condición de superdotación intelectual y por tanto, requieren atención educativa de acuerdo a sus necesidades específicas, de manera tal, que las experiencias educativas formales e informales constituyan acciones proactivas que faciliten el desarrollo de sus aptitudes individuales, y no sean una barrera para su crecimiento educativo integral.

Bajo estas consideraciones, el plan de intervención psico-pedagógico sugerido tiene un enfoque multifactorial, según el cual la noción de inteligencia está conformada por múltiples componentes, relativamente independientes entre sí, pero que son fundamentales para el desarrollo óptimo del niño y niña con superdotación.

Partiendo de la importancia que tienen los diferentes ámbitos de desarrollo del ser humano y la importancia de trascender la concepción monolítica de la inteligencia, la propuesta educativa está apoyada en el planteamiento básico de Gagné (1995); por cuanto este representa un panorama integral para la intervención con niños y niñas con superdotación; incluyendo e interrelacionando otras áreas fundamentales para el desarrollo integral de los educandos.

El autor considera cinco dominios básicos de aptitudes a través de los cuales se manifiestan las habilidades ligadas a la superdotación, a saber: intelectual, creativo, socio-afectivo, sensomotor y un quinto ámbito denominado por él como percepción extrasensorial -ético-moral-.

Bajo este enfoque se establecen correlaciones entre los diferentes ámbitos y se logra adscribirlos a conductas específicas y observables, con lo cual, se facilita la elaboración de programas y herramientas para la intervención pedagógica, el seguimiento y la valoración adecuada de los niños y niñas con superdotación.

Para efectos de la propuesta planteada en la I etapa del proyecto de investigación, el dominio intelectual será entendido como cognitivo-intelectual, a fin de ir más allá de la connotación academicista que se ha dado al término "intelectual", incorporando los aspectos propios del procesamiento mental de la información, aspectos consustanciales a toda noción de inteligencia, y por ende de superdotación.

Igualmente, el dominio de percepción extrasensorial, será entendido en este contexto como aquellas aptitudes que no dependen de los sentidos físicos (oler, gus- 
tar, oír...), sino de la dotación innata particular, denominada por Zohar y Marshall como la "Inteligencia espiritual" propiciadora del sentido de justicia, verdad, integridad, y moralidad; por lo tanto, se definirá para efectos de esta propuesta el ámbito extrasensorial como el dominio ético-moral, a fin de abarcar bajo este concepto, un rasgo muy importante, pero poco investigado como característica asociada a la condición de superdotación. Es decir, se integrará la parte subjetiva del niño y niña, su mundo de valores, de emociones y de deseos; áreas que tienen un valor fundamental en su desarrollo integral.

Además de lo anterior, es importante señalar que la incorporación del ámbito familiar, es parte fundamental de esta propuesta psico-pedagógica, porque el aprendiz es muy vulnerable a lo acontecido en su entorno familiar, en este debe ser aceptado y comprendido, gozando de la estimulación y el confort afectivo que le facilite el desarrollo y manifestación de su condición de superdotación con libertad.

La participación institucional: educadores/as y/o personal de apoyo adquieren un papel protagónico en el círculo educativo porque son los/as responsables del desarrollo educativo formal del alumnado, de donde se desprende la responsabilidad de proveer respuestas pedagógicas de acuerdo a las aptitudes y necesidades educativas particulares del educando con alta dotación, para su óptimo desarrollo y la propiciación de aprendizajes, experiencias, disciplina y práctica de habilidades, indispensables para la manifestación posterior de talentos específicos.

Esta propuesta Educativa de Enriquecimiento Extracurricular y su implementación, PEEEI, como ya se indicó, constituye una alternativa psicopedagógica oportuna que tiene su soporte en los resultados obtenidos en la investigación antes referida a cargo de la autora de esta propuesta.

\section{Problema de investigación}

Partiendo de una realidad nacional desesperanzadora para la niñez con alta dotación y sus padres, al no poder acceder a una educación con equidad para sus hijos/as se manifiesta irremediablemente el problema de inopia o privación de alternativas de intervención educativa validadas para la educación de la nińez con superdotación, se ha abordado el mismo, mediante la construcción e implementación de la propuesta planteada en el apartado anterior (PEEEI), que de comprobarse su efectividad científico-académica, esta formaría parte de una respuesta efectiva al problema y debería compartirse en espacios de interés educativo en el tema.

\section{Justificación}

La Propuesta Educativa de Enriquecimiento Extracurricular y su implementación presentada, pretende contribuir con el Derecho de todos los niños y niñas, a una educación con calidad, tornando impostergable el desarrollo de incursiones educativas que propicien la evolución de la educación hacia la consecución de una igualdad real de oportunidades educativas; proporcionando a cada aprendiz espacios pedagógicos, recursos mediáticos y apoyo humano que respondan efectivamente a sus necesidades educativas especiales (n.e.e.), tal como se ratificó ańos atrás 
en la Conferencia Mundial de Salamanca, España (1994) y como ya se mencionaba en las políticas educativas costarricenses referidas a la integración educativa de todo el alumnado desde hace casi 30 décadas, (MEP. 1987, p. 5).

Por consiguiente, se debe avanzar hacia propuestas teórico-prácticas que fundamenten transformaciones educativas sistémicas, porque es sabido que aunque los aprendices altamente dotados están incluidos en las instituciones educativas regulares; no están integrados en espacios educativos democráticos dada la evidente desigualdad de oportunidades respecto a la "normalidad"; propiciando así, dificultades de adaptación escolar u otros problemas asociados a su condición de superdotación.

Por lo tanto, se necesitan proposiciones innovadoras que enriquezcan la oferta educativa y respondan a la diversidad educativa sistémica de la sociedad contemporánea, una educación que esté en función de las condiciones particulares del sujeto; respetando las diferentes capacidades de aprendizaje y su entorno, lo cual se traduce en oportunidades educativas solidarias y en igualdad de oportunidades.

Además, la escasez de profesionales capacitados en el tema y la carencia de recursos adecuados en el sistema educativo, se suma a la rigidez curricular de las estructuras educativas vigentes, como ya se mencionó, dando como resultado su borrosidad escolar de los niños y niñas con superdotación.

Los aspectos señalados, constituyen barreras que es preciso derribar para poder avanzar y crear entornos educativos solidarios, que den respuesta a la diversidad educativa, y en el caso específico que nos ocupa, atender las necesidades educativas derivadas de las diferencias en las capacidades, complejidad y ritmos de aprendizaje de la población educativa con superdotación.

\section{Objetivos}

La construcción y validación de la PEEEI, tiene la intensión de generar o confirmar información y productos de valor educativo en un campo específico, como resultado de las interacciones con educadores/as, padres de familia y educandos con alta dotación ambientes muy heterogéneos que participan activamente en la II etapa de la investigación, en espacios de aprendizaje formales e informales, pretendiendo que la propuesta constituya una opción efectiva de intervención psicopedagógica y social, bajo un enfoque multidimensional evidenciado en el planteamiento de los siguientes objetivos:

\section{Objetivos generales:}

1. Plantear una propuesta de enriquecimiento extracurricular dirigida a la atención integral de nińos/as con superdotación en edades tempranas, sustentando la misma en sus necesidades educativas particulares desde una dimensión integral.

2. Implementar la propuesta educativa de enriquecimiento extracurricular dirigida a la atención integral de niños/as con superdotación en edades tempranas, mediante su aplicación en el campo. 


\section{Objetivos específicos:}

1.1. Realizar un programa de atención individual para cada aprendiz con superdotación, basado en sus necesidades educativas específicas en las áreas del desarrollo intelectual, creativo, socio-afectivo, sensomotriz y ético-moral mediante el cual se optimice su desarrollo educativo.

1.2 Construir una propuesta de intervención psico-pedagógica integral para los educandos con superdotación en la que se abarque la dimensión familiar y escolar, mediante la planificación de acciones específicas con estos actores del proceso educativo.

2.1. Implementar la propuesta de intervención psico-pedagógica integral, incluyendo en el proceso a los nińos/as con superdotación, padres y docentes a cargo, propiciándoles experiencias de aprendizaje, apoyo y estimulación, mediante la atención pedagógica directa y la orientación y capacitación a docentes y padres de familia como colaboradores.

2.2. Lograr que los/as niños/as, padres y educadores/as del estudio participen activamente en un proceso de interacción social mediante una CV.

2.3. Evaluar los resultados de la propuesta de intervención educativa en diferentes líneas de acción incluidas, que son:

a) La eficacia de la propuesta de intervención psico-pedagógica individual a través del progreso cuantitativo observado en c/u de las áreas intervenidas al trabajar con los educandos,

b) La eficacia de la propuesta de intervención pedagógica planteada, a través del nivel de comprensión y cambio de actitud de los padres de familia acerca de la condición de su hijo/a y el nivel de compromiso y apoyo adquirido,

c) La eficacia de la propuesta de intervención pedagógica planteada, a través del logro de los objetivos trazados y logrados con el personal docente a cargo.

d) La participación de todos los/as actores/as en la CV,

e) La conformación de una asociación de padres de nińos/as con alta dotación y talentos en Costa Rica.

\section{Hipótesis}

Los educandos con alta dotación logran un desarrollo psico-pedagógico de acuerdo a sus capacidades cuando son estimulados en las diferentes áreas de su desarrollo educativo y cuentan con el apoyo familiar y escolar.

\section{Aspectos metodológicos}

Como se ha señalado, esta es una investigación cuasiexperimental compuesta por dos etapas:

1. La elaboración de una propuesta teórica,

2. La implementación de la propuesta en el campo. 
Se concibió con fundamento en las necesidades educativas y socio-afectivas deslindadas de los resultados obtenidos en la evaluación diagnóstica de la muestra representativa de educandos con superdotación -14 niños/as- en edades tempranas entre 4 y 6 años, 6 niños y 8 niñas, que se encuentran ubicados en diversas regiones del país; a saber San José, Pérez Zeledón. Alajuela, Pacayas de Cartago, Guápiles y Guanacaste, inmersos en las aulas de los jardines infantiles, al tiempo de su valoración diagnóstica.

\section{Etapa:}

Proyecto de una Propuesta Educativa de Enriquecimiento Extracurricular, esta se sistematiza bajo los siguientes aspectos metodológicos:

\section{La determinación de los y las participantes:}

Para la realización de la propuesta se considerarán 3 grupos de participantes:

a) Niños y niñas con superdotación.

b) Padres y madres de familia.

c) Docentes y/o personal de apoyo educativo.

\section{Definición de las líneas de trabajo:}

\section{a) Ámbito individual:}

a. 1) Intervención psico-pedagógica directa desde las fortalezas.

a. 1.1) Interacción entre iguales mediante una comunidad virtual (C.V).

\section{b) Ámbito familiar:}

b. 1) Apoyo individual.

b. 2) Capacitación formal.

b. 3) Interacción social (C.V).

b. 4) Inducción para consolidar una asociación de padres de niños/as con superdotación.

\section{c) Ámbito escolar:}

c. 1) Asesoría por parte de un facilitador calificado.

c. 1. 1) Capacitación docente.

c. 1. 1. 1) Interacción social (C.V).

\section{Etapa:}

Una vez definidas las variables de la propuesta, se procede a su implementación en el campo; esta será realizada por 2 facilitadores cualificados en el tema de la atención a nińos/as con alta dotación, que trabajarán con los/as diferentes actores/as 
en su contexto. En cuanto a los educandos, se les dividirá en 2 subgrupos de 7 niños/ as cada uno, desarrollando un programa de atención individual semanal, por un período de 6 meses aproximadamente, de la siguiente manera:

\section{a) Atención psicopedagógica individual:}

- La intervención educativa con c/u de los educandos se orienta a la estimulación y maximización de las aptitudes y capacidades cognitivo-intelectuales, creativas, afectivas,... mediante el desarrollo de acciones psicopedagógicas específicas basadas en las n.e.e. individuales, este proceso es facilitado por un/a profesional calificado/a.

- Entrenamiento técnico y participación en una comunidad virtual interactiva, (CV) donde son fortalecidas sus capacidades particulares en las áreas intervenidas (desempeño socio-afectivo, intereses, valores, autoestima...).

b) Ámbito familiar: aquí se desarrollan acciones claramente delimitadas, para promover el empoderamiento y autogestión de los padres de familia, para la búsqueda de soluciones que procuren el desarrollo integral de sus hijos/as.

b. 1) Apoyo directo y capacitación formal:

- Acompañamiento y orientación sobre las preocupaciones externadas acerca de los hijos/as,

- Sesiones presenciales y/o virtuales (comunidad virtual - CV -), que faciliten procesos de aprendizaje que les habiliten como agentes activos para la estimulación de la superdotación de sus hijos/as y la búsqueda de oportunidades educativas eficaces.

b. 2) Conformación de una asociación de padres/madres de niños/as con superdotación, sin fines de lucro. trabajo.

c) Ámbito escolar: este ámbito concentra sus esfuerzos en dos líneas de

c. 1) Capacitación docente: incluye la aplicación de un plan específico de capacitación para los docentes y el personal de apoyo que participe en el proyecto. Este se realizará mediante actividades presenciales y/o virtuales; propiciando el aprovechamiento de las tecnologías de la comunicación $(\mathrm{CV})$ informándoles sobre aspectos fundamentales de la niñez con superdotación y sus requerimientos educativos.

c. 2) Asesoría y acompańamiento directo:

-Visitas, asesorías y acompañamiento psico-pedagógico a los/as docentes y al personal de apoyo encargados/as del proceso educativo regular de los niños y las nińas seleccionados en el estudio.

Los resultados obtenidos con la implementación de esta propuesta, se totalizarán mediante el registro cuantitativo de las conductas o respuestas y cumplimiento de los objetivos planteados en los programas de intervención individual con los/ as niños/as, la asistencia y participación de padres y docentes en las actividades de 
capacitación formal y las participaciones de los/as niños/as, padres y docentes en la comunidad virtual $(\mathrm{CV})$. En el proceso de evaluación se hará uso de las escalas likert como método de evaluación sumativa de aplicación individual.

\section{Presentación y análisis de los resultados}

La investigación consistió en dos etapas: el planteamiento teórico y la implementación de la propuesta (PEEEI); los datos que se presentan seguidamente corresponden a los resultados obtenidos en el proceso de aplicación de la misma entre los/ las 14 nińos/as, sus padres y educadores/as a cargo.

\section{Ámbito de trabajo individual:}

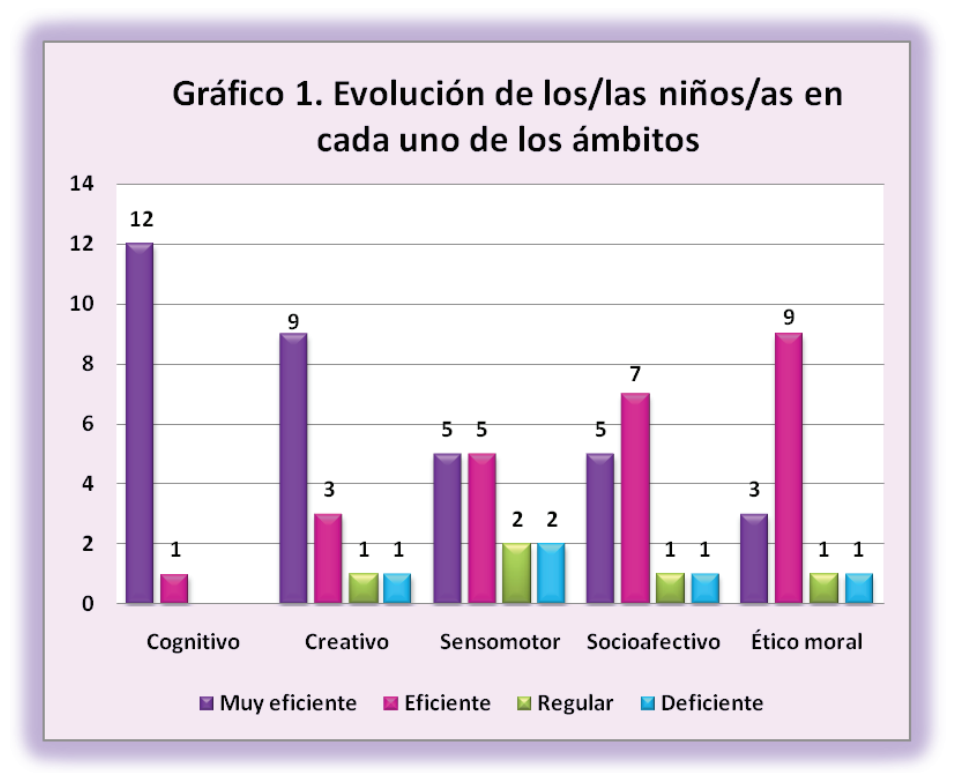

\section{Cognitivo Intelectual:}

El 100\% de los/las niños/as con alta dotación que participaron en el programa de enriquecimiento educativo en el ámbito cognitivo-intelectual, respondieron en forma efectiva y muy efectiva a la intervención individual en esta área; por lo tanto, se confirma como muy exitoso.

De la muestra representada, 12 de los trece niños(as) mostraron más de 10 veces la conducta, actitud o destreza que se deseaba alcanzar, por lo que su nivel cognitivo-intelectual mostró un avance muy eficiente,

1 niño manifestó un avance eficiente, realizando de 6 a 10 veces la conducta o destreza que se deseaba alcanzar, 
1 niña no participó en las actividades del ámbito intelectual por desmotivación debida a problemas intrafamiliares y escolares; por lo tanto, no fue cuantificada en la muestra representada en este ámbito.

\section{2. Ámbito creativo:}

El nivel de respuesta al programa de atención creativa fue exitoso. En términos generales los educandos mostraron un avance muy eficiente y eficiente en el $86 \%$ de los casos, estos resultados lo definen como positivo.

9 niños(as) mostraron más de 10 veces las actitudes o destreza que se deseaban estimular con alto grado de eficiencia, 3 niños(as) presentaron un avance eficiente mostrando la conducta creativa esperada, entre 6 y 10 veces.

1 nińo tuvo un avance regular, al mostrar las conductas creativas esperadas de 2 a 6 veces y 1 niña tuvo avance deficiente mostrando la conducta creativa esperada 2 o menos veces.

\section{3. Ámbito sensomotor:}

El gráfico 1 en el ámbito sensomotor muestra que el 72\% de los niños/as del programa obtuvieron resultados muy eficientes (5) y eficientes (5). 2 niños(as) mostraron un avance regular. 2 niñas mostraron un avance deficiente, una de ellas por ausencia en la participación al programa y la otra niña prefirió recibir clases de violín en ese período. Aunque los resultados en términos generales son positivos, se evidenció una situación frecuente en la niñez con alta dotación, al manifiestar cierta torpeza en el área sensomotora, porque en muchos casos piensan y procesan la información con mayor agilidad que la de sus destrezas y coordinación motriz fina.

\section{4. Ámbito Socio-afectivo:}

El gráfico 1, indica que en el $\mathbf{8 6} \%$ de los casos los resultados fueron muy satisfactorios. 5 niños(as) mostraron un avance muy eficiente. 7 niños(as) mostraron un avance eficiente. 1 niño tuvo un avance regular y 1 niña no participó del todo.

$\mathrm{Al}$ analizar los resultados anteriores, se estableció una correlación importante entre el desarrollo socio-afectivo individual del educando y el tipo de soporte que recibe de docentes y padres de familia; los resultados obtenidos evidencian que aquellos/ as niños/as que tuvieron poca aceptación, comprensión y seguridad afectiva por parte de padres y docentes (2) tuvieron un avance en el ámbito socio-afectivo menor que el de sus compañeros/as, tal es el caso de una niña que se inhibió de participar en el programa por carecer de la seguridad intrafamiliar necesaria en ese periodo y por el rechazo manifestado por su maestra de grado.

\section{5. Ámbito Ético Moral:}

En este ámbito del desarrollo, se destaca una participación muy eficiente y eficiente con respecto al plan de intervención para maximizar valores ético-morales en el $\mathbf{8 6} \%$ de los niños y nińas atendidos. 3 de los niños(as) mostraron un avance muy eficiente, 9 niños(as) mostraron un avance eficiente. 1 niño mostró avance regular y 1 niña no participó del todo. En términos generales se confirma su alto grado de sen- 
sibilidad mediante las respuestas positivas/constructivas ante situaciones planteadas, que involucraron la presencia de valores ético-morales, relevantes.

\section{Ámbito familiar:}

En el ámbito familiar se trabajó la mayor parte del tiempo con las madres de los educandos, desarrollando actividades de apoyo personal y capacitación formal, también, se les aplicó un cuestionario realizado específicamente para retroalimentarles a partir de los resultados. Varias de las sesiones de trabajo tuvieron por objetivo orientarles y motivarles para conformar la I asociación de padres de niños/as con superdotación en el país.

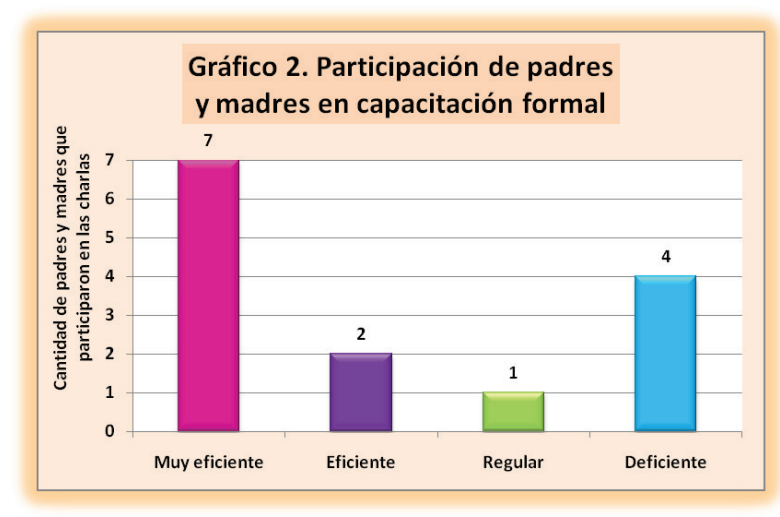

\section{Análisis del gráfico \#2.}

El $65 \%$ de los padres aproximadamente participaron de manera muy eficiente y eficiente en los procesos de acompańamiento y capacitación con profesionales calificados, un $7 \%$ tuvo una respuesta regular y el $28.5 \%$ de los padres respondieron en forma deficiente.

$\mathrm{Al}$ analizar los resultados se evidenció que un 35.5\% de los padres respondió negativamente; al indagar las causas, estos señalaron dificultades laborales y desconocimiento de la verdadera condición de sus hijos/as (asocian superdotación con niveles académicos excepcionales); sin embargo al finalizar el proceso la actitud de la mayoría había cambiado. 


\section{Ámbito Escolar:}

En el ámbito escolar, los/las educadores recibieron o tuvieron acceso a las actividades del programa dentro de las escuelas donde laboran con los/las niños/as con superdotación. Se trabajó específicamente haciendo observaciones para retroalimentarles y en capacitación sobre el tema fuera de clase, se les aplicó un cuestionarios para maestros/as, realizado específicamente para su retroalimentación por parte de los facilitadores del programa.

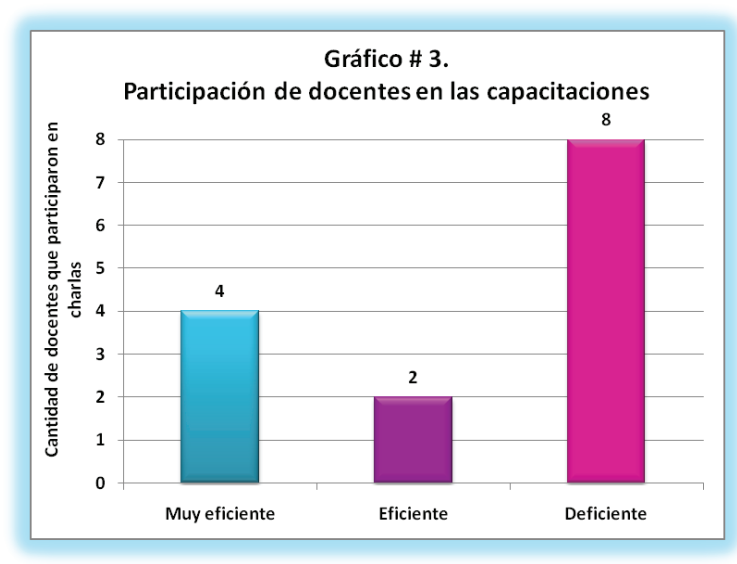

4 docentes participaron con un alto grado de compromiso y resultados muy eficientes, en el proceso de capacitación, 2 de manera eficiente y 8 de forma deficiente. Con base en los resultados obtenidos, se podría inferir que un mayor porcentaje $(57 \%)$ de educadores/as, poseen poca sensibilidad y compromiso hacia la atención educativa requerida por los educandos con alta dotación. Sólo un 43\% respondió al proceso de información, capacitación y acompańamiento brindado por los facilitadores del proceso.

\section{Participación de: niños/as, padres de familia y educadores/as en la comunidad virtual}

Ánálisis comparativo: niños/as, padres y docentes en la cv.

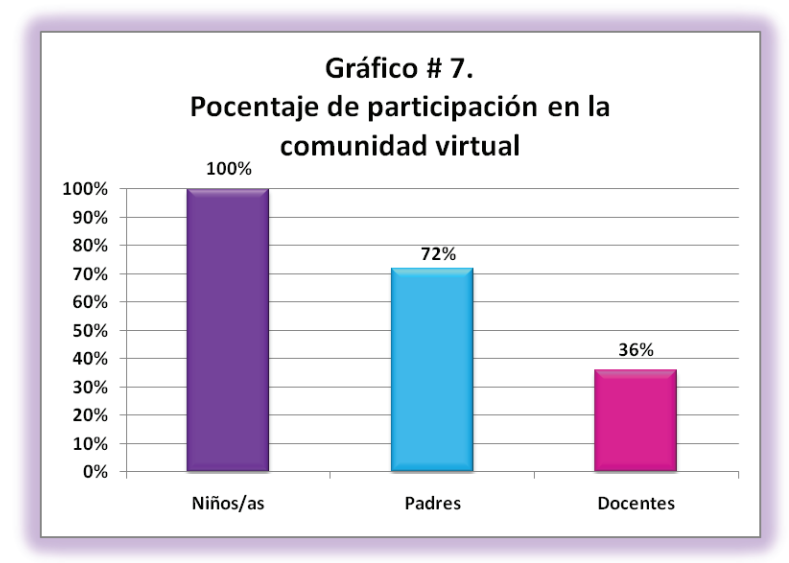


El cuadro anterior representa un análisis comparativo del nivel de respuestas o participación mostradas entre los educandos, padres de familia y sus profesores/as a través del espacio cibernético de la comunidad virtual, (CV).

La barra color púrpura representa la participación de los educandos en la CV, el $\mathbf{1 0 0} \%$ respondió positivamente a sus uso como medio de aprendizaje, expresión de intereses e interacción social.

La barra color turquesa representa la participación e interacción social de los padres en la CV, el $\mathbf{7 2} \%$ mostró un alto grado de interés en participar, pero variables como la brecha digital (varios nunca habían accedido al uso del computador), la poca experiencia en el uso de la tecnología como recurso de aprendizaje e intercambio y el hecho de no contar con la herramienta en su casa, contribuyó para que su participación no fuera óptima, pero a pesar de eso se esforzaron por participar y la mayoría se identificaron con la propuesta a.

En cuanto al profesorado, se registró un nivel bastante significativo de desinterés en apoyar la iniciativa con un $\mathbf{3 6} \%$ de participación, pudiéndose interpretar estos resultados como una falta de sensibilidad y compromiso hacia las necesidades psico -pedagógicas de este alumnado, a pesar de contar con el soporte de los facilitadores.

$\mathrm{Al}$ interpretar los resultados comparativamente, se evidencia la urgencia de trabajar en la sensibilización, y formación docente para atender a la niñez con superdotación desde sus particularidades educativas, pues en su caso el acceso al recurso tecnológico no fue una limitante, como si lo fue para algunos padres de familia; sin embargo, su participación en la CV fue muy baja. Por tanto, se debe seguir trabajando para integrar a padres y profesorado en procesos de intervención educativa integral, porque sus actitudes $(+/-)$ y su nivel de apoyo afecta significativamente el desarrollo psico-pedagógico del niño/a. (Ej. El caso de la niña que se inhibió de participar en el programa de atención psicopedagógica porque paralelamente mostraba serios problemas socio-afectivos en el contexto: escuela-hogar).

\section{Conclusiones}

Los resultados desprendidos al implementar la PEEEI en los ámbitos de intervención: cognitivo-intelectual, creativa, motriz, afectivo, entre otros) han confirmado la correlación existente entre las fortalezas del educando con superdotación y su nivel de respuesta ante la acción psico-pedagógica guiada por los facilitadores/as del proceso.

El estudio realizado evidencia una directa relación entre niño/a-escuela-hogar, por lo tanto, se deberían abarcar estos elementos integralmente si se quieren tener óptimos resultados en la intervención psicopedagógica, aunque se trate de nińos/as con alta dotación.

Queda confirmado mediante la experiencia teórico-práctica presentada, que los educandos con alta dotación en nuestros entornos latinoamericanos carecen de oportunidades pedagógicas que respondan adecuadamente a sus necesidades reales, pues tanto en los centros educativos como en las familias se observó ausencia de 
información sobre el tema, carencias en cuanto formación educativa para abordar los casos específicos que fueron identificados en una acción precedente, desorientación, frustración e ignorancia de servicios a los cuales acudir o recursos que aprovechar.

Tanto, padres de familia como profesorado desconocen en gran medida los derechos de este conglomerado a una educación con calidad y sobre todo los docentes no muestran adecuados niveles de compromiso con la educación de esta niñez.

La utilización de las herramientas tecnológicas como recursos pedagógicos y mediático constituye una fortaleza educativa, si son bien aprovechados, estos recursos son vistos por los educandos con beneplácito y entusiasmo, mostrando un óptimo nivel de respuesta ante su uso.

Se concluye, que la mayoría de padres y docentes aún le dan mucha credibilidad a los métodos convencionales para enseñar-aprender y no aprovechan los medios virtuales, ignorando el potencial de las herramientas tecnológicas si se utilizan adecuadamente en los procesos educativos de los educandos.

El tema de la intervención psico-pedagógica con niños/as altamente dotados/ as y talentosos/as es incipiente en nuestro entorno, queda casi todo por hacer desde nuestras esferas de acción educativa.

\section{Alcances}

- Validación de una propuesta específica de Intervención Psico-Educativa basada en las n.e.e de la niñez con superdotación en edades tempranas y que puede aplicarse en diferentes contextos.

- Contar con una opción psico-pedagógica con sustento científico-académico para compartirla en simposios, charlas, medios escritos, congresos dando a conocer los resultados de la I y II etapa de la investigación (1 ańo de trabajo).

- Aportes de material educativo en el tema de la superdotación infantil para fortalecer la página web elaborada en Costa Rica sobre el tema: www.uned.ac.cr/ altadotacionytalentos.

- Haber colaborado en el cambio de perspectiva de un grupo de padres de nińos/ as con superdotación que continuarán el camino con ellos/as como facilitadotes de su desarrollo integral.

- Las instituciones educativas -docentes- en las cuales se realizó la implementación de la propuesta con uno o más niños/as logró cambios significativos en la comprensión de lo que significa la atención a la diversidad educativa.

- Consolidación de la primera asociación de padres de niños/as con superdotación en Costa Rica. 


\section{Referencias}

CAMPOS, A. (1997). Sobre los fundamentos y estructura lógica de los proyectos de investigación social. San José: Universidad de Costa Rica.

GAGNÉ, F. (1995). From giftedness to talent: a developmental model and its impacto $n$ the language of the field. In: Roeper Review, December. Traducción (1997) en Ideacción 10, Valladolid, España: Centro Huerta del Rey.

GAGNÉ, F. (2007-2008) Construyendo talentos a partir de la dotación: Breve revisión del MDDT 2.1. Montreal, Canadá: Universidad de Québec.

HERNÁNDEZ, M.; FERNÁNDEZ, C.; BAPTISTA, L. (2006). Metodología de la Investigación. (3Ed). México: Editorial Mc Graw Hill.

Las variables de investigación. Desde <http://cmapspublic2.ihmc.us/rid=1191529176578_326104003_6792/ variables_de_investigacion.pdf> Extraído el 13 de octubre de 2010.

MORALES, K. (2007). Ideacción \# 27. Investigación basada en la Aplicación del Test Screening con base empírica para la Identificación Temprana de niños/as de 4,5 y 6 años con superdotación intelectual. Validación en Costa Rica. ISSN 1695-7075. Valladolid, España: Centro Huerta del Rey.

PRADO, M.L. (2008). Talento e superdotação: problema ou solução. Curitiba, Brasil: Editorial IBPEX.

SALDAÑO, O.H. (n.f.). Tesis de grado. Metodología de la investigación. Extraído el 13 de octubre de 2010 desde http://www.mailxmail.com/curso-tesis-investigacion/variables-concepto

SÁNCHEZ, F. (2008). Psicología social. Madrid, España: Editorial Mc Graw Hill.

UNESCO (2004). La educación de niños con talento en Iberoamérica. Santiago, Chile: Editorial Trineo S.A.

ZOHAR D.; MARSHALL I. (2001) Inteligencia Espiritual. Barcelona, España: Janés Editores.

\section{Nota}

${ }^{1}$ Morales, K. (2007). Investigación basada en la Aplicación del Test Screening con base empírica para la Identificación Temprana de niños/as de 4,5 y 6 años con superdotación intelectual. Validación en Costa Rica. Ideacción \# 27. ISSN 1695-7075. Valladolid, España.

\section{Correspondência}

Krissia Morales Chacón - Apartado 1894-2050, San Pedro de Montes de Oca - Costa Rica.

E-mail:kmoralescr@yahoo.com

Recebido em 14 de abril de 2014

Aprovado em 11 de julho de 2014 
